\title{
Radioprotection et installations nucléaires : réformer pour plus d'efficacité et de transparence
}

Le service central de protection contre les rayonnements (SCPRI) joue un rôle capital dans le contrôle des installations nucléaires de notre pays. Je rappelle brièvement que ses domaines d'action sont l'hygiène générale dans le domaine de la radioprotection des populations, l'hygiène professionnelle des travailleurs exposés, l'agrément des installations médicales et le contrôle des installations nucléaires. II a donc une mission fondamentale pour la radioprotection de la population et des travailleurs du nucléaire.

\section{Une perception brouillée}

Dans le premier rapport de l'Office parlementaire en 1990, mon collègue Sérusclat, en charge de cette partie, notait la difficulté de voir l'autorité chargée de la radioprotection reconnue par l'ensemble des associations de protection de l'environnement comme par l'ensemble des médias. En 1991, à plusieurs reprises, le SCPRI a encore été au centre de critiques violentes formulées par la presse et différentes organisations de protection de l'environnement.

Pour être objectif, il faut noter que la perception du SCPRI à l'étranger et dans les organisations internationales est bonne. II faut noter, aussi, le dévouement inlassable et le sens aigu du service public tant de ses responsables que de ses fonctionnaires.

Deux dossiers à l'origine des problèmes et polémiques : le suivi dosimétrique des travailleurs et les mesures de radioactivité dans l'environnement.

\section{Le suivi dosimétrique des travailleurs : des déficiences critiquables.}

C'est à l'automne 91 que la presse se fait l'écho de confessions de cadres d'EDF qui "ne péuvent plus se taire" et qui sont présentés comme dénonçant les écarts très significatifs entre les doses collectives recensées par le SCPRI et celles recensées par l'exploitant. A partir d'une erreur - involontaire ou non - sur des tailles d'échantillon, le soupçon est jeté sur l'autorité chargée de la radioprotection. Cette question est grave et je m'y suis penché avec attention. En effet, les doses annuelles reçues 
par le personnel d'EDF représentent environ $20 \%$ du total et celles reçues par le personnel extérieur $80 \%$, principalement lors des arrêts de tranche. Le personnel des entreprises sous-traitantes est particulièrement vulnérable car, souvent, les travaux de faible qualification en zone exposée leur sont confiés.

Déjà en 1990, le rapport de l'Office parlementaire posait la question de savoir s'il faut mettre en place des systèmes de dosimétrie nominative. II proposait : "la création de systèmes centralisés nationaux interconnectés en un seul réseau informatisé européen doit être rapidement développée !"

Deux lacunes graves doivent être dénoncées :

- Le laxisme et l'inertie de l'exploitant : ce dernier est chargé d'une part de mettre en œuvre son propre système de suivi des doses absorbées et, d'autre part, d'assurer la coordination des mesures prises par lui et par l'ensemble des employeurs et l'échange des informations entre ceux-ci et lui-même. La clé du sérieux de la dosimétrie des employés des sous-traitants et travailleurs intérimaires est dans les mains de l'exploitant. Par ailleurs, alors que les moyens techniques de mesure et de télétraitement existaient depuis plusieurs années, force est de constater qu'il a fallu attendre 1991 pour voir l'exploitant mettre en place son système national DOSIMAT. Je rappellerai fermement aux cadres en mal de confessions médiatiques des exploitants que le contrôle du port par tous les travailleurs extérieurs des instruments d'identification et de suivi leur incombe. D'autre part, ils ne doivent pas oublier non plus que, sur le site, les ISR (ingénieurs "Sûreté radioprotection") font office d'inspecteurs du travail.

- La lente mise en place de la réglementation pour la surveillance radiologique des travailleurs : il n'a pas fallu moins de 39 mois pour que la circulaire rendant applicable le décret du 6 mai 1988 soit publiée. Cette carence de l'Etat est inadmissible dans un domaine sensible où le règlement, la loi doivent protéger les plus faibles et les plus exposés, en l'occurrence les travailleurs sous-traitants et intérimaires.

\section{Les mesures de radioactivité de l'environnement}

A intervalles quasi réguliers, telle ou telle association dénonce dans la presse le silence des autorités devant les lacunes ou les déficiences du contrôle officiel ou la découverte de décharges plus ou moins clandestines ou oubliées, etc. Les spécialistes répondront que ces "trouvailles" n'en sont pas, que l'interprétation des résultats est fausse, etc. II n'empêche, ces informations jettent un doute ou, à défaut, pour le moment, du discrédit sur les organismes officiels, donc sur l'Etat lui -même.

Sans entrer dans l'analyse des arguments développés par les deux parties, sur la présence ou non de plutonium dans les sédiments du Rhône, aux abords de Super-Phénix, force est de constater que 
l'argumentation officielle vient après coup. II m'a semblé que la publication, avec les marges d'incertitudes, des résultats du point zéro radioécologique, par radioélément, aurait permis de voir immédiatement l'évolution des teneurs en éléments radioactifs. La consultation du réseau 3614 MAGNUC, ne donne pas les résultats d'analyses pour les émetteurs alpha et l'actualisation des données est parfois déficiente. De plus, il est difficile de comparer tant les méthodes de prélèvements que les méthodes d'analyses et, par conséquent, aussi les résultats, rien ne pouvant être identifié comme équivalent.

C'est pourquoi je recommande que les méthodes utilisées pour la détermination du point zéro soient les plus performantes du moment et que soient définis les paramètres normatifs des mesures effectuées dans l'environnement pour garantir la comparabilité des résultats. Ainsi, sera levé l'obstacle au pluralisme des mesures de radioactivité.

\section{Objectifs d'une réforme du SCPRI}

Partant de l'analyse d'une situation réelle et d'une image incertaine, les objectifs sont clairs : accroître l'efficacité de l'autorité chargée de la radioprotection, reconnaître celle-ci comme indiscutable tant par les exploitants que par les médias et l'opinion publique. J'inclus dans ma réflexion le point suivant : lorsqu'il y a contestation de mesures de radioactivité dans l'environnement par les organismes officiels ou lorsqu'il y a contestation des résultats de mesures de doses collectives reçues par les travailleurs, c'est, à travers ces organismes, l'autorité de l'Etat qui est mise à mal. Ce n'est pas acceptable. Tout le schéma de réforme que j'ai élaboré repose sur ce postulat : la protection de la santé des français vis-à-vis des risques nucléaires fait partie intégrante des missions de l'Etat. Ce choix de base est un principe intangible : la responsabilité de l'Etat est directement en cause à la fois au niveau de la réglementation et au niveau du contrôle du respect de cette réglementation.

Céci passe par une présence accrue du ministère de la santé dans le chamo des problèmes de radioprotection. même si l'habitude de laisser le SCPRI en "électron libre" lui a évité d'être en première ligne. Ceci passe par une présence accrue du ministère du travail qui a bien en charge les conditions de travail. Ceci passe par une implication du ministère de l'environnement pour tout ce qui concerne les mesures de radioactivité dans l'environnement. Cette dernière présence permet de faire la synthèse entre les problèmes de protection de la santé et de protection de l'environnement.

Le schéma que j'ai élaboré transpose au domaine de la radioprotection le schéma d'organisation du ministère de l'industrie avec la DSIN (Direction de la sûreté des installations nucléaires). Je propose donc de créer une direction de la protection contre les rayonnements ionisants (DPRI) au ministère de la santé, directement rattachée au ministre et 
mise à disposition, en tant que de besoin, du ministre du travail. La DPRI est chargée de la définition de la réglementation et de son application (voir plus loin le détail des recommandations). Le SCPRI devient l'appui technique de cette direction. II est chargé du contrôle de la radioprotection des travailleurs et du contrôle des installations nucléaires. Par analogie avec la DSIN, la DPRI se voit dotée de quatre groupes permanents chargés de la conseiller et de constituer un réseau de compétences dont elle puisse bénéficier dans sa tâche de définition et de mise en œuvre de la réglementation. Les quatre groupes permanents traitent respectivement des questions suivantes :

- épidémiologie et étude générale des effets sur la santé des rayonnements ionisants,

- santé et sécurité des travailleurs des installations nucléaires de base et des populations concernées par ces installations,

- santé et sécurité des travailleurs des autres installations utilisant des sources permanentes ou temporaires de rayonnements ionisants,

- effets des rejets de radioéléments sur l'environnement.

Je terminerai par deux missions confiées à la DPRI, sur lesquelles je souhaite insister :

- Le développement d'études épidémiologiques : dans notre pays, cette lacune est patente. II n'y a que de timides essais qui, faute de moyens, n'ont pu être pérennisés. Cela signifie aussi que l'on n'a pas pu tirer d'enseignements significatifs. Une étude limitée a été conduite en Angleterre, près de Sellafield. De même, en 1991, une étude a été publiée aux Etats-Unis et porte sur les taux de mortalité par cancer observés parmi le personnel du Laboratoire du Département de l'énergie à Oak Ridge. L'étude générale du National cancer institute aux Etats-Unis n'apporte pas de preuve d'une corrélation entre cancer et installation nucléaire. Nous ne pouvons, nous ne devons pas rester en dehors de ce champ d'étude des risques induits. Nous devons promouvoir ces études pour avancer dans leur connaissance, compte tenu de l'importance de notre parc nucléaire, ce qui peut donner signification aux résultats observés.

- Le développement du pluralisme des mesures sur des bases claires : le décret du 9 mai 1988 a prévu une méthode d'harmonisation des mesures de radioactivité de l'environnement et des denrées consommables, ainsi qu'une procédure de délivrance de certificats de qualification aux laboratoires qui souhaiteraient être homologués par l'administration. La pratique n'a pas suivi la théorie et les mécanismes prévus n'ont pas permis l'émergence du pluralisme souhaité et si nécessaire à la transparence. C'est pourquoi je propose que l'agrément soit donné par le ministre de la santé, après avis d'une commission d'agrément composée du directeur de la DPRI et des présidents des 4 groupes permanents. Ainsi pourra se mettre en place un réseau de laboratoires agréés qui pourront assurer des expertises pour le compte des commissions locales d'information ou d'associations ou de collectivités. 
L'ensemble de ces propositions, étayées sur une analyse lucide et sans complaisance de la situation, me paraît une architecture cohérente, claire, compréhensible, susceptible :

- de renforcer l'autorité de l'Etat, à travers l'autorité chargée de la radioprotection ;

- de mieux identifier cette autorité en séparant ce qui concerne la réglementation elle-même et son analyse technique ;

- d'organiser le pluralisme et la transparence des analyses et de l'information dans le domaine de l'environnement.

La persistance de contestations sporadiques mais continues me conforte dans l'idée qu'il n'y a pas d'autre issue crédible. Il est temps que le gouvernement s'engage, c'est son intérêt, celui de l'ensemble de la communauté nucléaire et la condition d'une information de qualité, jugée comme telle par nos concitoyens parce que pluraliste.

\section{Claude BIRRAUX}

Docteur ès sciences, député de Haute-Savoie, rapporteur de l'Office parlementaire des choix scientifiques et technologiques pour la sûreté nucléaire,

5, av. de la République, 74103 Annemasse Cedex, et 126, rue de l'Université, 75355 Paris. 